\title{
Purely absorptive bistability in double-ring cavities
}

\author{
P. M. Mejías, ${ }^{*}$ R. Martínez-Herrero, and E. Bernabeu \\ Departamento de Optica, Facultad de Ciencias Físicas, Universidad Complutense, 28040 Madrid, Spain
}

(Received 8 July 1985)

\begin{abstract}
A novel type of optical bistable configuration, namely the so-called double-ring (DR) device, is presented. The stationary solutions in the purely absorptive case for a homogeneously broadened atomic system are derived. In the mean-field limit it has been pointed out that use of a DR arrangement enables us to reduce both the length of the sample of the nonlinear medium and the response time of the device. Linear stability analyses have shown that the position and the length of the unstable regions of a given hysteresis cycle can be controlled, to a great extent, by means of the two new parameters of our DR scheme. Finally, it has been remarked that, in order to observe selfpulsing, the purely absorptive DR system may present a similar behavior to that offered in the dispersive single-ring case.
\end{abstract}

\section{INTRODUCTION}

As is well known, optical bistable devices are expected to be practically useful in the near future working as optical memories, switches, and amplifiers. ${ }^{1}$ From a tecknological point of view, most recent efforts have concentrated principally on the designing of miniaturized optical bistable devices that might work faster than semiconductor gates at similar switching energies. However, certain theoretical limits have been established. In fact, since the value of the absorption coefficient $\alpha$ is limited by practical material requirements, it appears that miniaturization and fast-switching operations can be attained only if the length $D$ of the sample of the nonlinear medium is sufficiently reduced. Moreover, from the semiclassical Maxwell-Bloch equations applied to the purely absorptive case in a ring cavity

$$
\alpha D / T>8
$$

has been obtained ${ }^{2}$ as a condition which should be fulfilled in order to obtain a bistable behavior. In this expression, $T$ is the transmissivity of the mirrors. Therefore, since $D$ is limited by transit-time considerations, in order to achieve bistability a small enough parameter $T$ should be used. Thus the value of $T$ constitutes an ultimately practical limitation.

On the other hand, the stability features of any hysteresis cycle should also be analyzed in detail. In fact they either limit the performance of the bistable device or improve its technological applications. Thus it has been shown ${ }^{3}$ that under suitable conditions a part of the hightransmission branch of the $S$-shaped curve of transmitted versus incident light becomes unstable, although in some cases the system may exhibit a self-pulsing behavior which can be used to convert $\mathrm{cw}$ light into pulsed light. In this connection Lugiato suggested ${ }^{4}$ that in order to observe self-pulsing, the dispersive case may offer definite advantages over the purely absorptive situation. In fact, in the dispersive case one finds regions in which no stationary solution is stable, which never occurs in the purely absorptive regime for the usual ring-cavity configuration analyzed in the literature. ${ }^{2-4}$ Thus, for such dispersive systems, the possibility of precipitation to a low- transmission state can easily a priori be excluded. Also note that, for purely absorptive systems inside a ring cavity in the mean-field limit, both the bistability curve and the unstable regions remain fixed for fixed values of the cooperativity parameter $C=\alpha D / 2 T$ and of the length $L$ of the cavity.

In this paper we present a novel type of optical bistable device which enables us to employ more accessible practi-

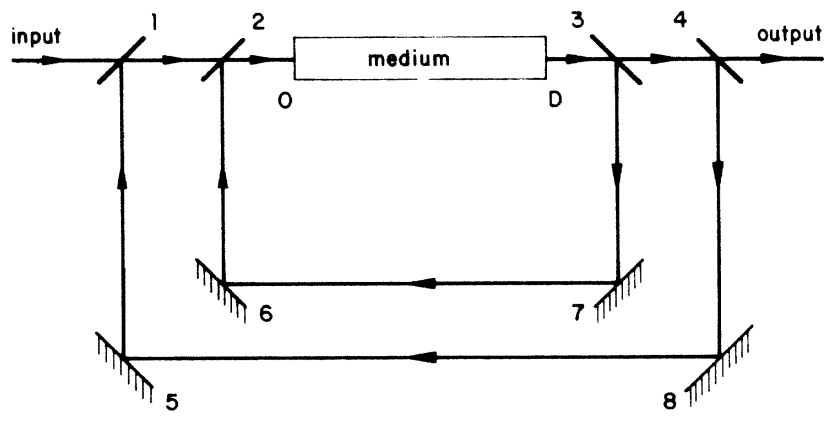

(a)

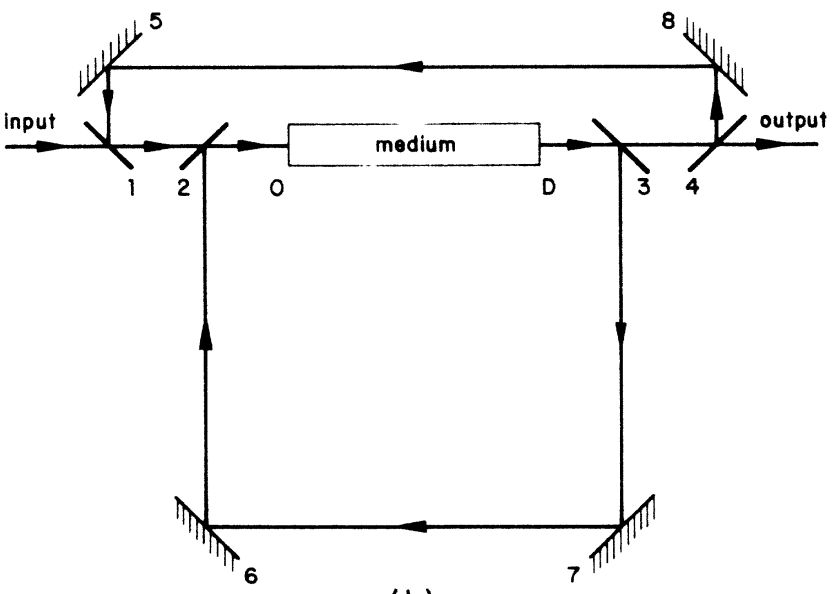

(b)

FIG. 1. Two alternative double-ring cavity schemes. In both configurations, external $(1,4)$ and inner $(2,3)$ mirrors have transmissivities $T_{0}$ and $T$, respectively, whereas mirrors 5-8 have $100 \%$ reflectivity. The total lengths of the outer- and inner-ring cavities are $L_{0}$ and $L$, respectively. (a) $L_{0}>L$, so that $M \equiv L_{0} / L>1$. (b) $L_{0}<L$, which implies $M<1$. 
cal values of the transmissivity and may control, to a great extent, the position and the length of the unstable regions of its hysteresis cycle. Such a device (see Fig. 1) considers the usual single-ring (SR) cavity scheme as a "basic cell" around which another external ring-cavity configuration is arranged. ${ }^{5}$ In the present work we restrict ourselves to the study of the purely absorptive case, although our analyses will be extended in subsequent papers to those cases in which no atomic or cavity resonance exists.

In Sec. II we determine the exact steady-state equation for optical bistability in a double-ring (DR) cavity. The mean-field approach is introduced and some interesting consequences are derived from a comparison with the corresponding expressions for the SR case. In Sec. III we investigate the stability of the stationary solutions in the mean-field limit. It will be shown that in order to observe self-pulsing, a purely absorptive DR system may present a similar behavior that offers the dispersive SR case. Moreover, by means of two control parameters (the transmissivity $T_{0}$ of the external mirrors and the total length $L_{0}$ of the outer cavity) the instabilities of the hysteresis cycles may be controlled. Finally, Sec. IV summarizes the principal conclusions of this work.

\section{STEADY-STATE ANALYSIS}

Let us consider the schematic diagram represented in Fig. 1. A sample of length $D$ containing a system of two-level atoms is placed inside a DR arrangement as shown in Fig. 1. As is well known, if the incident field (assumed to be a plane wave) is resonant with the atoms and with the cavity, integration of the Maxwell-Bloch equations for the stationary case gives ${ }^{2}$

$$
\ln \left(\frac{F(0)}{F(D)}\right)+\frac{1}{2}[F(0)]^{2}-\frac{1}{2}[F(D)]^{2}=\alpha D,
$$

where

$$
F(0)=\frac{\mu}{\hbar\left(\gamma_{\perp} \gamma_{\|}\right)^{1 / 2}} E(0),
$$

and

$$
F(D)=\frac{\mu}{\hbar\left(\gamma_{\perp} \gamma_{\|}\right)^{1 / 2}} E(D),
$$

$E(0)$ and $E(D)$ being the slowly varying envelope of the monochromatic electric field at the input and output planes of the sample, respectively, $\mu$ is the modulus of the dipole moment of the atoms and $\gamma_{\|}$and $\gamma_{\perp}$ are the longitudinal and transversal atomic relaxation rates. For a perfectly tuned DR cavity it can be readily shown that the proper boundary conditions reduce to the following equation:

$$
E(0)=\left(T T_{0}\right)^{1 / 2} E_{I}+\left(R_{0} T+R\right) E(D),
$$

where $E_{I}$ is the incident field over mirror 1 and $R_{0}=1-T_{0}$. Then by defining

$$
x=\frac{\mu E_{T}}{\hbar\left(\gamma_{\perp} \gamma_{\|} T T_{0}\right)^{1 / 2}}=F(D),
$$

$$
y=\frac{\mu E_{I}}{\hbar\left(\gamma_{\perp} \gamma_{\|} T T_{0}\right)^{1 / 2}},
$$

where $E_{T}$ is the transmitted field amplitude (see Fig. 1), we can rewrite Eq. (5) as follows:

$$
F(0)=T T_{0} y+x\left(1-T T_{0}\right) .
$$

Finally, by combining Eqs. (8) and (2) we obtain

$$
\begin{aligned}
\ln \left[1+T T_{0}\right. & {\left.\left[\frac{y}{x}-1\right)\right] } \\
& +\frac{x^{2}}{2}\left\{\left[1+T T_{0}\left(\frac{y}{x}-1\right)\right]^{2}-1\right\}=\alpha D,
\end{aligned}
$$

which is the exact stationary relation between the transmitted field (proportional to $x$ ) and the incident field (proportional to $y$ ). Let us now consider the mean-field limit

$$
T \rightarrow 0, \alpha D \rightarrow 0, \quad \frac{\alpha D}{2 T} \equiv C \text { const } .
$$

In this approach the quantity $T T_{0}(y / x-1)$ is infinitesimal, so that Eq. (9) reduces to the expression

$$
y=x+\frac{2 C^{\prime} x}{1+x^{2}},
$$

where $C^{\prime} \equiv C / T_{0}$. Equation (11) is the state equation of the mean-field theory of optical bistability in a DR cavity. A number of interesting consequences may be derived from this expression. First of all, note that Eqs. (9) and (11) exactly coincide with those obtained for a SR cavity (see Ref. 2) if we make $T_{0}=1$ in our expressions. In fact, in the mean-field limit, $C^{\prime}$ plays the same role as the parameter $C$ in the SR situation. More specifically, the threshold bistability condition now becomes

$$
C^{\prime}>4 \text {, }
$$

which formally coincides with the corresponding condition for the SR case. We can refer to the product $T T_{0}$ as the "global" transmissivity of the DR device. Taking this into account the following result can be proven at once: Given any hysteresis cycle representing the bistable behavior of the system in a SR cavity, there exists an infinite collection of DR devices (having the same global transmissivity) which identically reproduces such hysteresis cycles. This situation is illustrated in Figs. 2-8: All bistability curves represented for the same value of $C^{\prime}$ identically coincide. Note that no stability analysis has been applied yet. In Sec. III it will be seen that the unstable regions of the hysteresis cycles of all the previously shown DR configurations present important differences.

On the other hand, the transmissivity $T_{0}$ of the upper exterior mirrors can be considered as a true external control parameter because it constitutes a supplementary degree of freedom in our DR system. In fact, for any value of $\alpha, D$, and $T$, an optical bistable behavior can be obtained by suitable choice of the value of $T_{0}$. Moreover, to work with smaller cavities it will suffice to work with smaller values of the independent parameter $T_{0}$ [cf. Eq. (12)]; but if the dimensions of the cavity are reduced, then 
the transit time also decreases. Therefore we may finally conclude that in a sense, a DR device can work faster than the usual SR devices.

\section{LINEAR STABILITY ANALYSIS}

To analyze the stability of the stationary solutions in the mean-field limit we shall apply the standard procedure followed, for example, in Ref. 4. Thus, let us first consider the Maxwell-Bloch equations for a homogeneously broadened atomic system

$$
\begin{aligned}
& \partial P / \partial t=(\mu / \hbar) E D-\gamma_{\perp} P, \\
& \partial D / \partial t=-(\mu / 2 \hbar)\left(E P^{*}+E^{*} P\right)-\gamma_{\|}(D-N / 2), \\
& \partial E / \partial t+c \partial E / \partial t=-g P,
\end{aligned}
$$

where $E(z, t)$ refers to the electric field, $P(z, t)$ is the macroscopic polarization, $D(z, t)$ is one-half the difference between the population of the lower and the upper level, $N$ the number of two-level atoms, $c$ the velocity of light in the vacuum, and $g$ a coupling constant given by

$$
g=4 \pi \omega_{0} \mu / V,
$$

$\omega_{0}$ being the frequency of the incident field and $V$ the volume of the atomic sample. For the sake of definiteness we shall analyze the case $\gamma_{\perp}=\gamma_{\|} \equiv \gamma$. Of course, our results can be extended in a straightforward way to the general case. Equations (13)-(15) should be completed with the corresponding equations for $E^{*}$ and $P^{*}$, which are the complex conjugates of Eqs. (15) and (13), respectively. ${ }^{6}$ Now let $E_{\mathrm{st}}(z), P_{\mathrm{st}}(z)$, and $D_{\mathrm{st}}(z)$ be one of the stationary solutions of Eqs. (13)-(15). If we introduce the deviations

$$
\begin{aligned}
& \delta F(z, t)=F(z, t)-F_{\mathrm{st}}(z), \\
& \delta P(z, t)=P(z, t)-P_{\mathrm{st}}(z), \\
& \delta D(z, t)=D(z, t)-D_{\mathrm{st}}(z),
\end{aligned}
$$

where

$$
F(z, t)=\frac{\mu E(z, t)}{\hbar \gamma},
$$

and linearize Eqs. (13)-(15) around the stationary solution, we obtain the following expressions:

$$
\begin{aligned}
& \frac{\partial \delta P}{\partial t}=\gamma\left(F_{\mathrm{st}} \delta D+D_{\mathrm{st}} \delta F\right)-\gamma \delta P, \\
& \frac{\partial \delta D}{\partial t}=-\frac{\gamma}{2}\left[\left(F_{\mathrm{st}} \delta P^{*}+P_{\mathrm{st}}^{*} \delta F\right)+\text { c.c. }\right]-\gamma \delta D, \\
& \frac{\partial \delta F}{\partial t}=-c \frac{\partial \delta F}{\partial z}-\frac{\mu g}{\hbar \gamma} \delta P,
\end{aligned}
$$

together with the complex conjugates of Eqs. (21) and (23) that, for brevity, we omit. The boundary condition for $\delta F$ now becomes (DR case)

$$
\begin{aligned}
\delta F(0, t)= & R_{0} T \delta F\left(D, t-\left(L_{0}-D\right) / c\right) \\
& +R \delta F(D, t-(L-D) / c),
\end{aligned}
$$

where $L_{0}$ is the total length of the outer cavity (see Fig. 1). For the sake of convenience we rewrite the linearized equations in a complex matricial form as follows:

$$
\frac{\partial v}{\partial t}=\widehat{M} v, \quad v=\left(\begin{array}{c}
\delta P \\
\delta P^{*} \\
\delta D \\
\delta F \\
\delta F^{*}
\end{array}\right)
$$

where the matrix elements of the operator $\widehat{M}$ are

$$
\begin{aligned}
& M_{11}=-\gamma=M_{22}, \\
& M_{12}=0=M_{15}=M_{21}=M_{24} \\
& M_{13}=\gamma F_{\mathrm{st}}=M_{23}^{*}, \\
& M_{14}=\gamma D_{\mathrm{st}}=M_{25}, \\
& M_{31}=(-\gamma / 2) F_{\mathrm{st}}^{*}=M_{32}^{*}, \\
& M_{33}=-\gamma, \\
& M_{34}=(-\gamma / 2) P_{\mathrm{st}}^{*}=M_{35}^{*}, \\
& M_{41}=-\mu g / \hbar \gamma=M_{52}, \\
& M_{44}=-c \partial / \partial z=M_{55} .
\end{aligned}
$$$$
M_{12}=0=M_{15}=M_{21}=M_{24}=M_{42}=M_{43}=M_{45}
$$$$
=M_{51}=M_{53}=M_{54} \text {, }
$$

As is well known ${ }^{4}$ the stationary field $F_{\text {st }}$ would be stable if and only if all the eigenvalues $\lambda$ of $\hat{M}$ have a negative real part. Hence the linear equation system we should solve is

$$
\begin{aligned}
& -\gamma_{p}+\gamma F_{\mathrm{st}} d+\gamma D_{\mathrm{st}} f=\lambda p, \\
& -\gamma p^{*}+\gamma F_{\mathrm{st}}^{*} d+\gamma D_{\mathrm{st}} f^{*}=\lambda p^{*}, \\
& -\gamma / 2\left(F_{\mathrm{st}}^{*} p+P_{\mathrm{st}}^{*} f+\text { c.c. }\right)-\gamma d=\lambda d, \\
& -\mu g / \hbar \gamma p-c \frac{\partial f}{\partial z}=\lambda f, \\
& -\mu g / \hbar \gamma p^{*}-c \frac{\partial f^{*}}{\partial z}=\lambda f^{*},
\end{aligned}
$$

together with the boundary condition (24). In Eqs. (27)-(31) we have denoted $p \equiv \delta P(0, z), d \equiv \delta D(0, z)$, $f \equiv \delta F(0, z)$, etc. Note that in the mean-field limit the stationary values become uniform in space, i.e., are independent of $z$. In order to solve the above equations we could try to find an explicit expression of $\delta F(z, t)$ that satisfies (24). It is easy to see that such a function is

$$
\delta F(z, t)=e^{h z} e^{\lambda t},
$$

with

$h=\frac{2 \pi i n-\ln \left[R_{0} T e^{-\left[\left(L_{0}-D\right) / c\right] \lambda}+R e^{-[(L-D) / c] \lambda}\right]}{D}$

( $n$ integers) .

Introducing this ansatz (32) into Eqs. (27)-(31) and taking the mean-field approach into account, one obtains after lengthy but elementary calculations that the values $\lambda$ are the solutions of the following equation: 


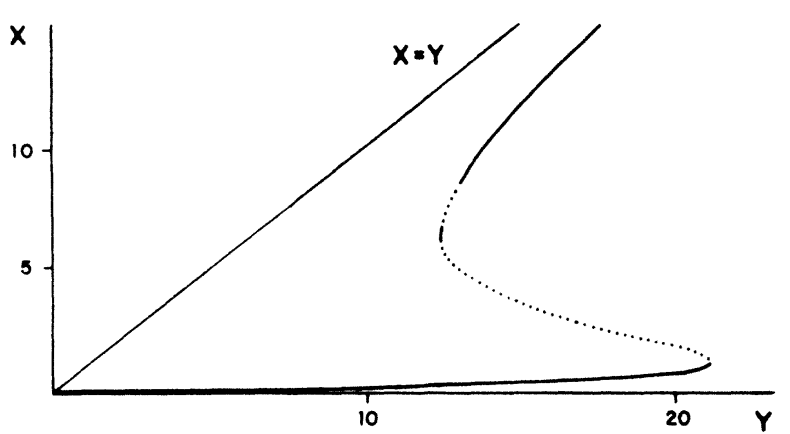

FIG. 2. Transmitted vs incident light for $C^{\prime}=20, \alpha D=0.05$, $T=0.00125, T_{0}=1$, and $c / L=\gamma$. The dotted part of the curve indicates the unstable regions.

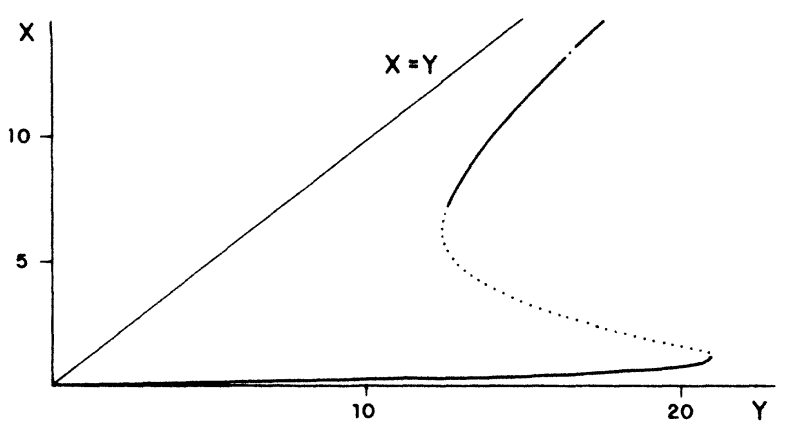

FIG. 4. Same as Fig. 3 but with $T=0.0125$ and $T_{0}=0.1$.

$$
R_{0} T e^{-L_{0} \lambda / c}+R e^{-L \lambda / c}=\exp \left[\frac{\alpha D \gamma}{1+x^{2}}\left[\frac{1}{\lambda+\gamma}-\frac{\gamma x^{2}(\lambda+2 \gamma)}{(\lambda+\gamma)\left[(\lambda+\gamma)^{2}+\gamma^{2} x^{2}\right]}\right]\right],
$$

where $x$ has been defined in (6). Since in the mean-field limit $T \rightarrow 0, \lambda$ can be approximately calculated from Eq. (34) by standard perturbative procedure at first order in $T$. Thus, if we write

$$
\begin{aligned}
& \lambda=\lambda_{0}+\lambda_{1} T \\
& a(\lambda, T) \equiv R_{0} e^{-L_{0} \lambda / c}=a(\lambda, 0)+\left.\frac{\partial a}{\partial T}\right|_{T=0} T, \\
& b(\lambda, T) \equiv R e^{-L \lambda / c}=b(\lambda, 0)+\left.\frac{\partial b}{\partial T}\right|_{T=0} ^{T,} \\
& \left.c(\lambda, T) \equiv \exp \left[\frac{\alpha D \gamma}{1+x^{2}} \mid \frac{1}{\lambda+\gamma}-\frac{\gamma x^{2}(\lambda+2 \gamma)}{(\lambda+\gamma)\left[(\lambda+\gamma)^{2}+\gamma^{2} x^{2}\right]}\right]\right]=c(\lambda, 0)+\left.\frac{\partial c}{\partial T}\right|_{T=0} T,
\end{aligned}
$$

and substitute these expressions into Eq. (34), after equating terms of the same order in $T$ one finally gets ${ }^{7}$

$$
\begin{aligned}
& \lambda_{0}=-i \alpha_{n}-K\left(1+\frac{2 C \gamma}{1+x^{2}} \frac{\gamma\left(1-x^{2}\right)-i \alpha_{n}}{\left(\gamma-i \alpha_{n}\right)^{2}+\gamma^{2} x^{2}}\right), \\
& \lambda_{1}=\frac{R_{0}}{R} \frac{\exp \left\{-\left[\left(L_{0}-L\right) / c\right] \lambda_{0}\right\}}{L / c+\left[\alpha D \gamma /\left(1+x^{2}\right)\right] Q},
\end{aligned}
$$

where

$$
\begin{aligned}
& \alpha_{n}=\frac{2 \pi c n}{L}(n \text { integers }), \\
& K=\frac{c T}{L},
\end{aligned}
$$

and

$Q=\frac{-\lambda_{0}^{4}-2 \gamma \lambda_{0}^{3}\left(2-x^{2}\right)+\gamma^{2} \lambda_{0}^{2}\left(7 x^{2}-6\right)+4 \gamma^{3} \lambda_{0}\left(2 x^{2}-1\right)+\gamma^{4}\left(3 x^{2}-1\right)}{\lambda_{0}^{6}+6 \gamma \lambda_{0}^{5}+\gamma^{2} \lambda_{0}^{4}\left(15+2 x^{2}\right)+4 \gamma^{3} \lambda_{0}^{3}\left(5+2 x^{2}\right)+\gamma^{4} \lambda_{0}^{2}\left(7+4 x^{2}+x^{4}\right)+2 \gamma^{5} \lambda_{0}\left(3+4 x^{2}+x^{4}\right)+\gamma^{6}\left(1+2 x^{2}+x^{4}\right)}$.

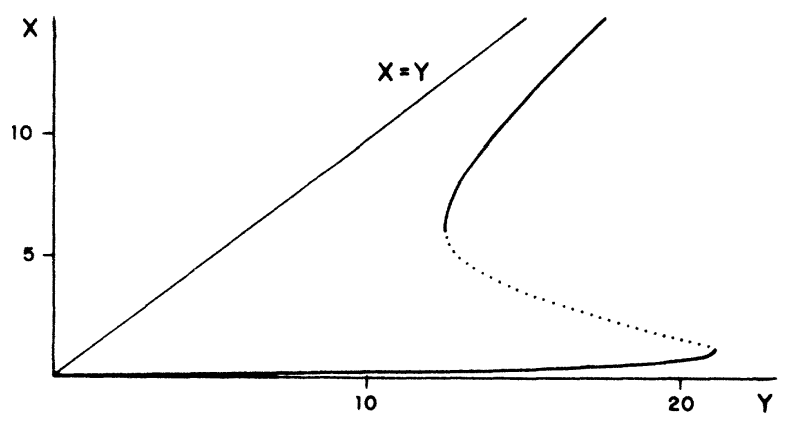

FIG. 3. Same as Fig. 2 but with $T=0.0025, T_{0}=0.5$, and $M=2.5$.

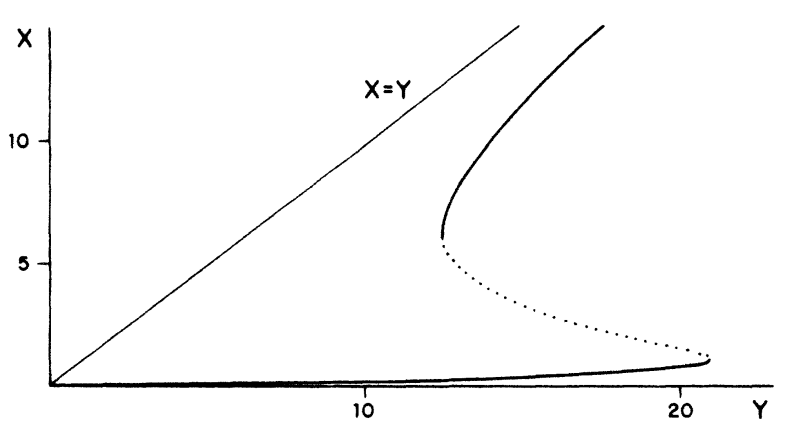

FIG. 5. Same as Fig. 3 but with $M=0.5$. 


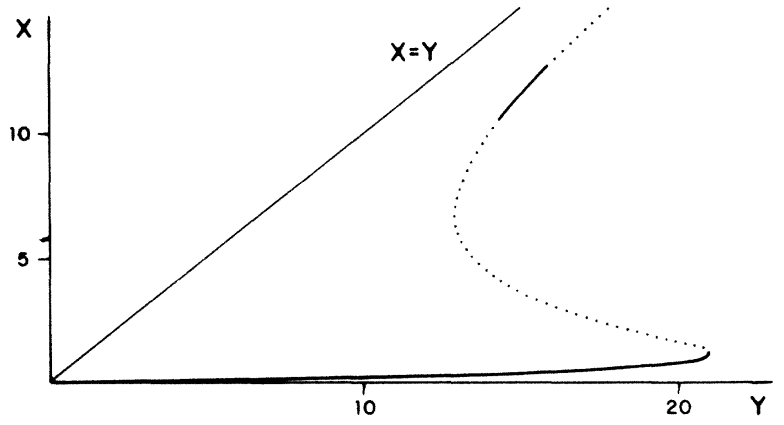

FIG. 6. Same as Fig. 2 but with $T=0.04, T_{0}=0.03125$, and $M=0.5$.

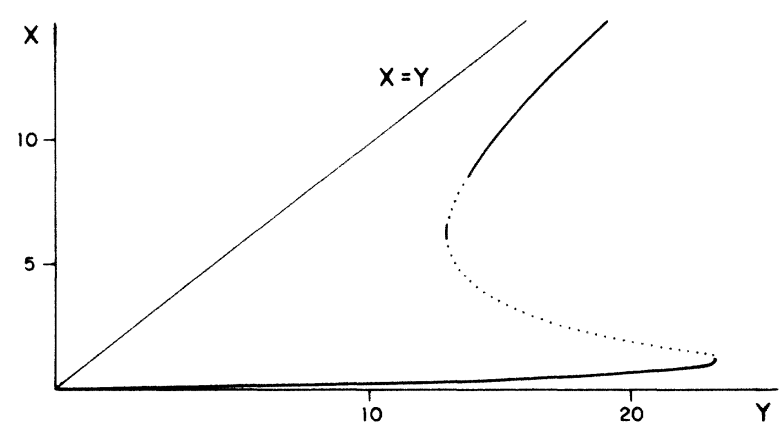

FIG. 7. Same as Fig. 2 but with $T=0.005, T_{0}=0.25$, and $M=2$.

Equation (39) takes the same form that the corresponding expression for $\lambda$ in the SR case [see Eq. (4.29) of Ref. 8]. In fact, putting $R_{0}=0$ in Eq. (40) our system reduces to the SR-cavity problem and we find the usual equation for the eigenvalues $\lambda .^{8}$ In Eq. (39) the index $n$ labels the modes of the cavity: Particularly $n=0$ refers to the mode which is resonant with the incident field.

Equations (35) and (39)-(43) are the general expressions that should be used for stability analyses. However, since in the mean-field limit $\alpha D \rightarrow 0$, Eq. (40) can be substantially simplified giving

$$
\lambda_{1} \cong \frac{R_{0}}{R} \frac{c}{L} e^{-\left[\left(L_{0}-L\right) / c\right] \lambda_{0}} .
$$

Thus our stability condition for a DR cavity finally becomes

$\operatorname{Re} \lambda=K\left(\frac{R_{0}}{R}\left\{\exp \left[K\left(\frac{L_{0}-L}{c}\right)\left[1+\frac{2 C \gamma A}{1+x^{2}}\right]\right] \cos \left[\left(\frac{L_{0}-L}{c}\right)\left[\alpha_{n}+K \frac{2 C \gamma B}{1+x^{2}}\right]\right)\right\}-1-\frac{2 C \gamma A}{1+x^{2}}\right)<0$

(for all $n$ integers),

where

$$
\begin{aligned}
& A=\frac{\gamma\left(1+x^{2}\right)\left[\alpha_{n}^{2}+\gamma^{2}\left(1-x^{2}\right)\right]}{\alpha_{n}^{4}+2 \gamma^{2} \alpha_{n}^{2}\left(1-x^{2}\right)+\gamma^{4}\left(1+x^{2}\right)^{2}}, \\
& B=\frac{\gamma^{2} \alpha_{n}\left(1-3 x^{2}\right)+\alpha_{n}^{3}}{\alpha_{n}^{4}+2 \gamma^{2} \alpha_{n}^{2}\left(1-x^{2}\right)+\gamma^{4}\left(1+x^{2}\right)^{2}}
\end{aligned}
$$

Condition (45) has been applied to some illustrative examples shown in Figs. 2-8. From the numerical analysis of these figures the following essential features can be inferred.

(a) First, note that in Fig. 2 we have chosen $T_{0}=1$. This implies that the curve plotted in this figure corresponds to the special case in which our DR cavity degenerates into the usual SR model. We see that, according to previous results, ${ }^{3}$ a part of the high-transmission branch (HTB) of the hysteresis cycle is unstable. Moreover, the central mode $n=0$ goes unstable on the part of the curve with negative slope whereas the off-resonance mode $n=1$ produces instability in the HTB.

(b) In Figs. 3 and 5 only the regions with negative slope become unstable (central-mode instability). Thus, by means of a suitable choice of the values of the parameters $M$ and $T_{0}$ in our DR system, the unstable regions throughout the HTB can be removed.

(c) Figures 4, 6, and 8 show that for sufficiently small values of $T_{0}$, instabilities in the HTB reappear. Moreover, we find situations in which no stationary solution is stable. For example, for $C^{\prime}=20, \alpha D=0.05, M=2.5$, and $T=0.04$, the whole bistability curve becomes unstable. As was pointed out by Lugiato ${ }^{4}$ this never occurs in the purely absorptive SR case. It should be noted that in both Figs. 4 and 6 the central mode becomes unstable throughout the first unstable part of the curve, whereas in correspondence to the second unstable region of the bistability plot the mode $n=2$ goes unstable. Also in Fig. 8, the resonant mode $n=0$ becomes unstable sooner for the values $1.1<x<6.9$, whereas side-mode instability $(n=1)$ appears in the rest of the unstable region.

(d) Figure 7 quite approximately coincides with Fig. $2 .^{9}$ However, their respective values of $T$ clearly differ. In fact, the transmissivity $T$ in Fig. 7 is four times the value

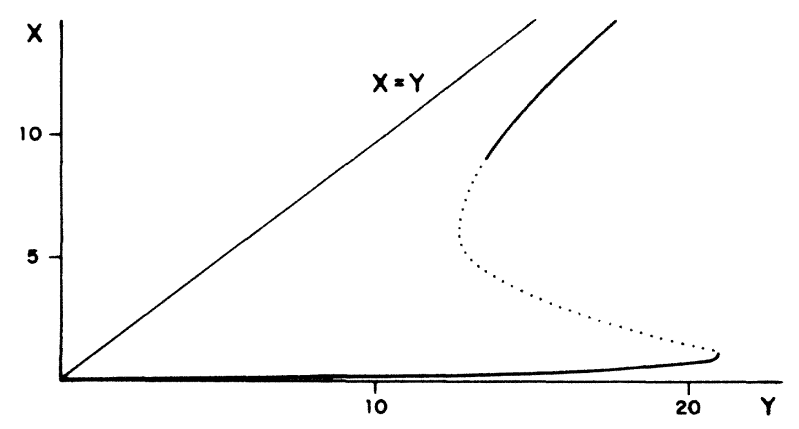

FIG. 8. Same as Fig. 7 but with $T=0.0125$ and $T_{0}=0.1$. 
of $T$ in Fig. 2. We thus see that our DR devices enable us to obtain, to a great approximation, the same bistability curves that are in the SR case but now with more accessible practical values of the transmissivities.

\section{CONCLUSIONS}

In the present work the main characteristic features of the so-called DR optical bistable devices have been discussed. In the purely absorptive case both the exact steady-state equation and the mean-field expression have been derived. From the comparison with the usual SR system we observe that our equations coincide with the corresponding expressions for the SR cavity if we write the product $T T_{0}$ instead of $T$ in the SR equations. In fact, there exists an infinite set of DR devices that exactly reproduce any given $S R$ hysteresis cycle. These results enable us (a) to obtain a bistable behavior for any value of $\alpha$, $D$, and $T$; (b) to reduce the length of the sample of the nonlinear medium; and (c) to work with faster devices. Such advantages follow from the use of a new control parameter, the transmissivity $T_{0}$ of the external mirrors.

On the other hand, detailed mean-field stability analyses have revealed that the unstable regions of the hysteresis cycle of a DR cavity strongly depend on the partic- ular choice of the transmissivities and on the value of the parameter $M \equiv L_{0} / L$. This fact clearly suggests that the unstable behavior of a given hysteresis cycle can be controlled, to a great extent, by using our DR optical device. Moreover, in order to observe self-pulsing it has been shown that the purely absorptive situation can offer similar advantages to those that present the dispersive SR case if a DR arrangement is used.

It has also been indicated which of the eigenvalues go unstable in the unstable regions. In general, the unstable eigenvalues in the SR case are different to those that appear in the DR configuration, although the central mode always becomes unstable on the part of the bistability curve with negative slope, at least in the examples tested by the authors. This is an interesting point which deserves further study in the future.

It also remains to apply our DR configuration to those systems having nonzero atomic or cavity detuning. This will constitute the next step of the present investigation.

\section{ACKNOWLEDGMENTS}

This work was supported by the Comision Asesora de Investigación Cientifica y Técnica (CAICyT) of Spain, under Project No. 2130/83.
"Present address: Departamento de Electricidad y Magnetismo (Optica), Universidad Nacional de Educación a Distancia (U.N.E.D.), 28040 Madrid, Spain.

'See, for example, E. Abraham and S. D. Smith, Rep. Prog. Phys. 45, 815 (1982), and references therein; see also S. L. McCall and H. M. Gibbs, in Dissipative Systems in Quantum Optics, edited by R. Bonifacio (Springer, Berlin, 1982), p. 93; and more recently the Proceedings of the Conference on Optical Bistability 2, edited by C. M. Bowden, H. M. Gibbs, and S. L. McCall (Plenum, New York, 1984).

${ }^{2}$ R. Bonifacio and L. A. Lugiato, Lett. Nuovo Cimento 21, 505 (1978).

${ }^{3}$ R. Bonifacio and L. A. Lugiato, Lett. Nuovo Cimento 21, 510 (1978); R. Bonifacio, M. Gronchi, and L. A. Lugiato, Opt. Commun. 30, 129 (1979).

${ }^{4}$ L. A. Lugiato, Opt. Commun. 33, 108 (1980).

${ }^{5}$ Double-ring situations have been recently considered by $\mathrm{K}$. Ikeda and M. Mizuno, Phys. Rev. Lett. 53, 1340 (1984). In this work the two-cavities scheme is presented as equivalent to the usual Fabry-Perot resonator and the respective equations of motion are reduced to double-delay differential equations.

${ }^{6} \mathrm{Although}$ in the present work we are analyzing the purely absorptive case, for the sake of generality, we shall consider in the calculations the complete set of five Maxwell-Bloch equations.

${ }^{7}$ There exist other two possible values of $\lambda_{0}$. See, R. Bonifacio and L. A. Lugiato, in Dissipative Systems in Quantum Optics, edited by R. Bonifacio (Springer, Berlin, 1982), p. 73. However, their real parts are strongly negative and cannot yield any unstable behavior.

${ }^{8} \mathbf{R}$. Bonifacio and L. A. Lugiato, in Dissipative Systems in Quantum Optics, edited by R. Bonifacio (Springer, Berlin, 1982), p. 61.

${ }^{9} \mathrm{As}$ in Fig. 2, the points of the curve with negative slope are unstable because the resonant mode is unstable, whereas sidemode instability $(n=1)$ appears on the second unstable region of the HTB of the plot. 